\title{
Screening for diabetic retinopathy-is the use of artificial intelligence and cost-effective fundus imaging the answer?
}

\author{
Aravind R. Sosale ${ }^{1}$
}

Published online: 28 February 2019

(C) Research Society for Study of Diabetes in India 2019

Vision loss among people with diabetes is a major problem. While most know the importance of evaluating a patient for complications, retinopathy screening gets left out by most treating physicians for want of expertise and training needed.

IDF atlas of 2017 [1] estimates that India has 72.9 million people with diabetes. Twenty percent of 24.4 million IGT add up to this number each year and along with 42.2 million undiagnosed cases quoted in the Atlas, takes the total close to 120 million. This figure is likely to double by 2045 as per experts. Really an epidemic that can be called the tsunami of diabetes!

IDF Atlas of 2017 also goes on to say that diabetic retinopathy (DR) is the leading cause of blindness and 1 out of every 3 patients has DR, and 1 out of 10 go on to develop "vision threatening' DR. International Association for Prevention of Blindness (IAPB) estimates 45 million to have vision threatening DR. Meta-analysis published in diabetes care [2] mentions that 1 out of 39 blind people had blindness due to DR and 1 out of 52 visually impaired persons has impaired vision due to DR. Figures are truly "eye opening" and frightening!

In India, prevalence of DR is close to $35.12 \%$ in people with diabetes after 5 years of duration of diabetes [3]. CINDI (chronic complications in newly diagnosed patients with type 2 diabetes in India) [4] published in 2014 reported that $6.1 \%$ of newly diagnosed type 2 patients have established DR at diagnosis. CINDI 2 in 2016 [5] looked at complications in newly detected T2 DM patients below the age of 40 years (young onset type 2 diabetes) and reported that $5.1 \%$ as the study population had established DR at diagnosis. These figures coming from national level survey data clearly tell us that India has a huge problem in terms of prevention of blindness due to DR.

Aravind R. Sosale

draravind@hotmail.com

1 Diacon Hospital, Bangalore, India

\section{Who should screen for DR?}

With hardly 1000 trained retina vitreous surgeons and about 3000 trained medical retina specialists in India, it is an impossible task to screen and treat all patients with retinal problems. So, the onus of screening all patients for DR falls on us physicians who treat them. As most treating physicians do not work in a set-up that has an in-house ophthalmologist, referral is a standard practice. Referring our cases to an ophthalmologist is not solving the problem as close to $70 \%$ of the patients do not reach them. Patients' vision is normal as DR is asymptomatic till the last stages and the seriousness of going blind never really hits a patient. Taking off from work, fixing appointment, additional cost, and the issue of not able to drive back after dilatation of the pupil are some of the reasons why those referred rarely stick to their retinal exam schedule.

Most diabetes treating doctors are not trained in direct or indirect ophthalmoscopy, a skill that needs training but hardly given during college days. A very small number of trained ophthalmologists specialize in vitreous-retinal surgery and medical retina as sub-specialties in ophthalmology is growing by the day. Not all eye specialists can treat DR, a fact which is not known to many physicians.

\section{Rationale for early detection of DR at physicians level}

There is an urgent need to improve methods for screening for DR as majority of the patients do not have symptoms till advanced macular edema or vitreous hemorrhage occurs. The efficacy of laser photocoagulation and/or vascular endothelial growth factor (VEGF) inhibitors in preventing visual loss from PDR and DME is well established in randomized trials. Early detection through screening programs and appropriate referral for therapy are important to preserve vision in individuals with diabetes. Most importantly, detection of DR helps a physician to tighten glycemic control, hypertension 
control, and control of dyslipidemia; to ensure smoking cessation; and to look for early markers of diabetic nephropathy. Most patients do take their condition more seriously after knowing that the complication has started.

Methods of screening for DR available at present include direct and indirect ophthalmoscopic examination by a trained doctor. Some centers with in-house ophthalmology department will also use "high-end" fundus cameras for capture of images and these cameras are also used to perform fundus fluorescein angiography for further evaluation. Since a treating physician in every city pan-India will not be able to invest so much for evaluation of DR, usage of cost-effective fundus cameras and tele-ophthalmology is an option.

In tele-ophthalmology, fundus images are sent to reading centers via Internet and trained doctors read the images and grade them as no DR, mild NPDR, mod NPDR, and severe NPDR and PDR. Diabetic macular edema (DME) is also graded as per internationally accepted norms. Unfortunately, India does not have "dedicated" centers for tele-ophthalmology unlike other countries. Very few hospitals in India have specialized set-ups as they have many satellite centers to cater to. Most doctors who work in this field are not trained retinal specialists but do this as a spare job. Experience, doctor-fatigue, image quality, pixel size, reader screen type, and Internet issues are some of the reasons for poor-quality grading. Medical errors are the 3rd leading cause of death in the USA which reported an interview in NEJM Catalyst attributable to cognitive errors and not because of bad clinicians [6].

The kappa statistic is frequently used to test interrater reliability in the field of tele-ophthalmology. Many studies [7, 8] have been done to assess agreement among a group of ophthalmic care providers, including ophthalmologists and trained nonphysician personnel, in the interpretation of one-field, two-field, and even three-field digital fundus images for diabetic retinopathy screening. There is only fair agreement among all readers. Most studies conclude by saying "The agreement of the evaluation of retinopathy grades did not correlate with periods of experience as ophthalmologists. The intra-rater agreement of the evaluation of retinopathy grades was $60-70 \%$. These results suggested that the reliability of the evaluation of retinopathy grades was not high among ophthalmologists." Even in some of our own studies (under publication), the Kappa score was only "fair" to "moderate" among five highly trained retina vitreous specialists while reading both two-field and three-field non-mydriatic and mydriatic images. Thus clearly, there is a need to look beyond tele-ophthalmology.

\section{Era of artificial intelligence to give us diagnosis}

Software algorithms that use artificial intelligence (AI) to aid in the diagnosis of retinal images are being developed worldwide [9]. Deep learning is considered as a fourth industrial revolution. It is based on learning features from the data. It processes large amount of data and extracts meaningful patterns from them. CNN (convolutional neural networks) algorithm teaches itself by analyzing a labeled training set of expert graded images and provides a diagnostic output.

Deep neural networks can detect subtle changes, patterns, or abnormalities that may be possibly at times be overlooked by human experts. The AI devices provide a screening decision without requiring an ophthalmologist to interpret the retinal images, hence can be used by physicians who may not normally be involved in eye care. Many AI softwares are available in the western world, but IDX is the only one to have the FDA approval in 2018. Most companies use cloud based "on-line" software support for making the diagnosis and hence need Internet support. India has the highest mobile usage in the world, but Internet still poses a problem. Medios is a Singapore-based company that has developed an AI-based solution and has partnered with Remidio Innovative Solutions Pvt. Ltd. that manufactures costeffective, FDA-approved fundus camera aptly named "Remidio FOP (Fundus on phone). The Medios AI based software works "Offline" and does not need Internet. iPhone fitted on the back of the camera has inbuilt DR detection software and diagnosis is made in less than 20 seconds. PDF report shows Lesions as heat maps indicating presence of DR that needs "referral". AI also checks the quality of the images before making the diagnosis and poor-quality images are discarded to avoid false reporting.

How good are the cost-effective cameras and AI support is the question that comes to all our minds? Among many costeffective cameras available in the Indian market, Remidio FOP is the only FDA-approved cost-effective camera. This camera has been compared with top end cameras like Topcon and Zeiss FF 450 cameras in separate studies $[10,11]$ and is found to have high sensitivity and specificity and has substantial agreement with conventional retinal photography. Moreover, studies conclude that "the rate of "ungradable" images was acceptably low and image quality was marginally better with Remidio FOP." This camera can be used as a "hand-held" device or can be mounted on a table top. Remidio FOP takes both mydriatic and non-mydriatic images of high quality. Most importantly any Lay person can be trained to take fundus images within a day.

Even though IDF recommends non-mydriatic fundus imaging for screening of DR, it is important for physicians to know that age of the patient, duration of diabetes, cataract, and pupil size can interfere with the quality of image in nonmydriatic fundus imaging. A single drop of tropicamide can be used to solve the problem. Chances of developing acute angle closure glaucoma are $0.006 \%$ as per most studies [12].

AI support for making the diagnosis with Remidio FOP has been tested in India extensively and many studies are ongoing. Fundus images of 301 patients with diabetes were run on the Medios Software at Dr. Mohan's Diabetes specialties Hospital at Chennai. The images were graded by an ophthalmologist 
according to the International Diabetic Retinopathy Classification System. The ophthalmologist was blinded to the diagnosis of the AI. The AI reported the images as REFER (mod, severe NPDR, PDR, and DME) or NO REFER (mild NPDR or no DR). The diagnosis of the AI was compared to that of the ophthalmologist. Results presented at APTOS July 2018 conference at Singapore (under publication) showed sensitivity for referable DR (mod, severe NPDR and PDR) as $96.6 \%$ (95\% CI 92.1-98.9) and for severe NPDR and PDR showed was $100 \%$ (95\% CI 94.8-100). In another study done by Prof. S Natarajan and colleagues from Aditya Jyot Eye Hospital, Mumbai was presented at the 2018 RSSDI conference (under publication). DR screening at Mumbai Municipal Corporation Dispensary done by his group used the AI to make the diagnosis and sensitivity for any DR was $85.2 \%$ and for referable DR (mod, severe PDR and DME) was $100 \%$.

Our own team at Diacon Hospital in association with Retina Institute of Karnataka has two large studies. First study took three images per eye (mydriatic) and compared the AI diagnosis with that of two senior retina vitreous surgeons. Total of six images per patient from 304 patients were analyzed and results are being presented at ATTD 2019 conference at Berlin during February. Sensitivity and specificity for referable DR (mod NPDR and more severe or the presence of DME) was 98.84 (95\% CI 97.62-100\%) and $86.73 \%$ (95\% CI 82.87-90.59\%). Sensitivity for sight threating DR (severe NPDR, PDR or the presence of DME) was $100 \%$. Sensitivity and specificity for any DR (mild, mod NPDR and more severe) was $86.78 \%$ (95\% CI 97.62$100 \%$ ) and $95.45 \%$ (95\% CI 82.87-90.59\%). This study is under publication.

To our knowledge, the largest study from India using an offline AI (SMART STUDY) on a portable cost-effective FOP camera is being presented at ADA 2019 at San Francisco from our team. The results of AI diagnosis using non-mydriatic retinal images of 900 patients are compared to the diagnosis of 5 ophthalmologists (considered as ground truth).

The results from various studies above help us as doctors in adopting newer technology in clinical care. The aim is to use technology that shows consistent, reliable, and accurate results across multiple studies.

\section{Conclusion}

Deep learning and artificial intelligence are here to assist us in screening for DR in all our cases. The Remidio FOP is portable, easy to use, FDA-approved, and captures non-mydriatic good quality images comparable to the best of the fundus cameras. Medios AI software for DR screening works offline on the iPhone, gives immediate reports, highlights lesions (heat maps) and produces PDF reports. Studies show that offline AI algorithm by Medios can be used for screening for diabetic retinopathy in primary care. The sensitivity of the Medios AI with mydriatic images exceeds the FDA-mandated superiority end points. This can open new doors to make DR screening more accessible. Larger and multi-center studies will further validate the results of our efforts.

The use of AI promises to reduce cognitive work load for physicians and ophthalmologists thus improving care, diagnostic accuracy while improving clinical and operational efficiency.

Who should bell the cat? We all as treating physicians must take responsibility for screening for DR.

If we do not screen for DR, our patients are likely to go blind.

Publisher's note Springer Nature remains neutral with regard to jurisdictional claims in published maps and institutional affiliations.

\section{References}

1. IDF ATLAS 8TH Edition. 2017: http://www.diabetesatlas.org/. Accessed on Feb 10th 2019.

2. Leasher JL, Bourne RRA, Flaxman SR, Jonas JB, Keeffe J, Naidoo $\mathrm{K}$, et al. Global estimates on the number of people blind or visually impaired by diabetic retinopathy: a meta-analysis from 1990 to 2010 . Diabetes Care. 2016;39(9):1643-9.

3. Gadkari SS, et al. Prevalence of diabetic retinopathy in India: the all India ophthalmological society diabetic retinopathy eye screening study 2014. Indian J Ophthalmol. 2016;64(1):38-44.

4. Sosale A, Prasanna Kumar KM, Sadikot SM, Nigam A, Bajaj S, Zargar AH, et al. Chronic complications in newly diagnosed patients with type 2 diabetes mellitus in India. Indian J Endocrinol Metab. 2014;18(3):355-60.

5. Sosale B, Sosale AR, Mohan AR, Kumar PM, Saboo B, Kandula S. Cardiovascular risk factors, micro and macrovascular complications at diagnosis in patients with young onset T2 diabetes in India: CINDI 2. Indian J Endocrinol Metab. 2016;20:114-8.

6. From the NEJM Catalyst Event "Future of care delivery: Relentless Redesign at Providence St. Joseph Health, January 19, 2017. https:// catalystqa.nejm.org/videos/business-case-promoting-wellness/

7. Ruamviboonsuk P, Ruamviboonsuk P, Ruamviboonsuk KT, Tiensuwan M, Yuttitham K, Thai Screening for Diabetic Retinopathy Study Group. Interobserver agreement in the interpretation of single-field digital fundus images for diabetic retinopathy screening. Ophthalmology. 2006;113(5):826-32.

8. Funatsu H, Yamashita H, Shimada H, Suzuki M, Ohashi Y. Reliability of evaluating grade of diabetic retinopathy. Nippon Ganka Gakkai Zasshi. 1993;97(3):396-402.

9. Raman R, Srinivasan S, Virmani S, Sivaprasad S, Rao C, Rajalakshmi R. Fundus photograph-based deep learning algorithms in detecting diabetic retinopathy. Eye (Lond). 2019;33:97-109.

10. Rajalakshmi R, Arulmalar S, Usha M, Prathiba V, Kareemuddin KS, Anjana RM, et al. Validation of smartphone based retinal photography for diabetic retinopathy screening. PLoS One. 2015;10(9): e0138285. https://doi.org/10.1371/journal.pone.0138285.

11. Sengupta S, et al. Sensitivity \& specificity of smart phone based retinal imaging for DR-A comparative study: ophthalmology retina 2018 by the American Academy of Ophthalmology. https://doi.org/ 10.1016/j.oret.2018.09.016.

12. Liew G, Mitchell P, Wang JJ, Wong TY. Fundoscopy: to dilate or not to dilate? The risk of precipitating glaucoma with mydriatic eye drops is very small. BMJ. 2006;332(7532). https://doi.org/10.1136/ bmj.332.7532.3. 